\title{
Intensive care medicine in 2050: the ICU in vivo
}

\author{
Can Ince $e^{1,2^{*}}$
}

() 2017 The Author(s). This article is an open access publication

When speculating on how an intensive care unit (ICU) might appear in 2050, the famous saying of the Noble laureate physicist Niels Bohr comes to mind: "prediction is very difficult, especially if it concerns the future." Nevertheless, the purpose of this paper is to speculate on the diagnostic developments that will define the landscape of the ICU in 2050. In doing so it should be noted that it is the view of a physiologist interested in intensive care medicine and not that of an intensivist. Although it is likely that the future will be dominated by personalized medicine [1], a concept will have to emerge which will be much more suited to the critically ill patient than its current understanding based mainly on genomics and biomarkers [2]. Personalized medicine will need to be more focused on the continuous assessment of the function of the various physiological compartments of the individual critically ill patient [3, 4]. We have termed this concept personalized physiological medicine, as a version of personalized medicine that is more closely related to the physiological needs of the critically ill patient [5]. The principles of personalized physiological medicine are defined by continuous assessment of the physiological reserve and frailty of the patient, the function of the various organ systems, the hierarchy and coherence of the cardiovascular system [6], and finally the integration of physiological variables and therapeutic interventions for control of organ function ([5]; Fig. 1). Personalized physiological medicine could be, in my opinion, the defining platform for the ICU of 2050 as it includes basic concepts from engineering for the control of complex systems [7]. To achieve this aim, continuous and quantitative measurements of essential physiological parameters that relate to organ and cellular function will be required using

\footnotetext{
*Correspondence: c.ince@erasmusmc.nl

${ }^{1}$ Department of Intensive Care, Erasmus MC, University Medical Center Rotterdam, 's-Gravendijkwal 230, 3015 CE Rotterdam, The Netherlands Full author information is available at the end of the article
}

tailor-made point-of-care technology. To achieve precision medicine, devices and sensors will have to be placed as close as possible to the organs and cells of the patient.

The specification for the real-time acquisition of essential physiological variables is dictated by the Shannon-Nyquist sampling theorem where sampling must occur at least at twice the rate of the fastest changing control variable [8]. This requirement is currently not met in the monitoring of several organ systems (e.g., kidney) because of intermittent sampling of essential variables (e.g., biomarkers). Achieving optimal measurement of such variables will require targeting biosensors to defined locations throughout the patient [9]. The parameters and location of these biosensors will be dictated by pathophysiology research leading the way for technology development for the ICU of 2050. The sensing of a physical variable or chemical substance in vivo requires biorecognition, signal transduction, and a detector. Sensors would be monitoring hemodynamic variables related to oxygen transport and utilization, the presence of biomolecules, parenchymal cell function, or gene transcription factors in various physiological compartments of the body. Even currently, biosensors are being manufactured at the submicron level (e.g., $[9,10])$ and it is expected that such nanodevices will be standard point-of-care in the ICU of the near future. Specialized novel materials are being developed including innovative synthetic materials such as nanocarbon tubes and synthetic biology in which biomolecules from biology such as antibodies and chemical and electrical sensors are incorporated into solid-state components $[9,10]$. The introduction of mobility to biosensors or theragnostic drug delivery devices by use of micro- and even nanomotors can allow homing of these sensors to target locations in the patient [11]. Sensors could also consist of swimming imaging capsules or implantable biosensors as part of organ assist devices to provide closed loop control or non-invasive externally located sensors that communicate with in vivo

\section{Springer}




\section{The Four Pillars of Personalized Physiological Medicine}

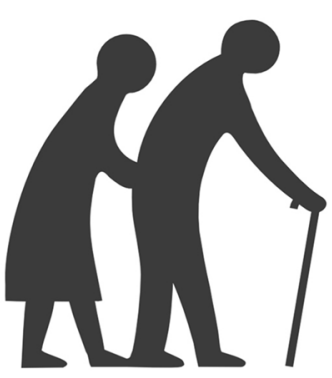

I. Frailty and Physiological Reserve

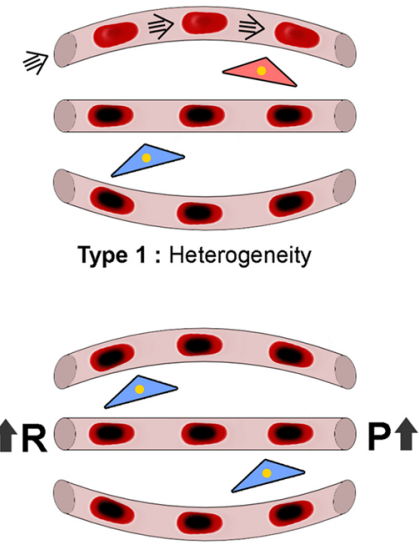

Type 3 : Constriction/Tamponade
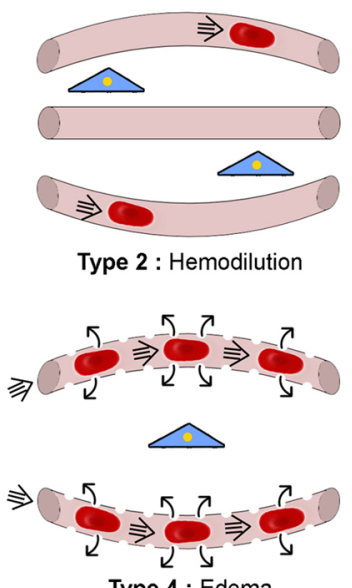

Type 4 : Edema
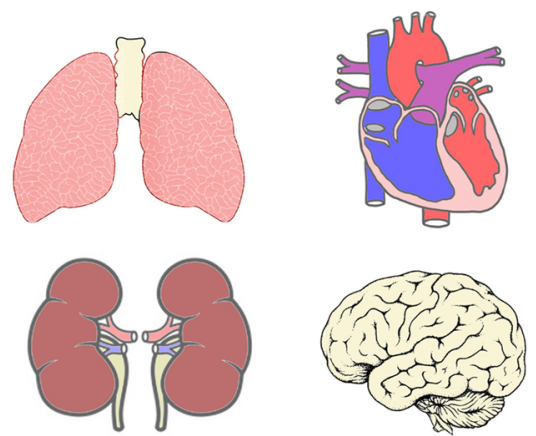

II. Organ Function Response to Therapy

\section{Hemodynamic Coherence}

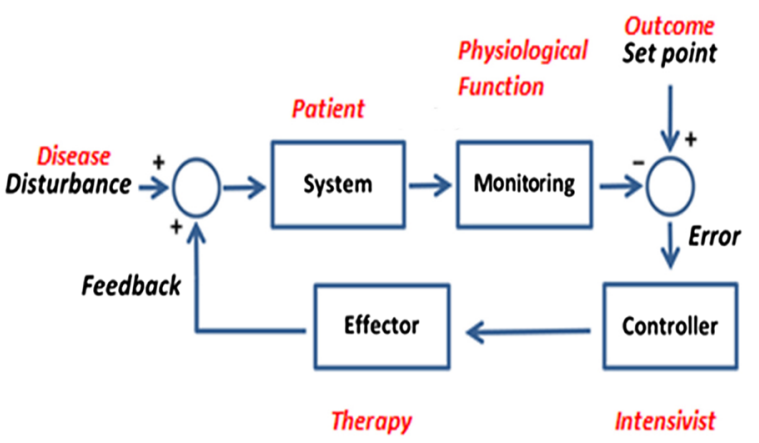

Fig. 1 The four pillars of Personalized Physiological Medicine required for tailor-made therapy of the individual critically ill patient are envisaged as consisting of: 1) assessment of the frailty, fitness and physiological reserve of the patient, 2) the continuous and quantitative functional assessment of the various organ systems, 3 ) the assessment of the coherence and physiological regulation of the different components of the cardiovascular systems from the systemic to the microcirculation to the cellular and subcellular functional structures that defines homeostasis (6) and finally, 4) an integration and feedback (7) of the defining physiological variables to drive therapy and provide clinical control of the patient. Adapted from (6)

sensors. Equally important in achieving the aims of the future ICU in vivo will be the development of telecommunication technology for the transmission of information between biosensors and outside the patient to a remote station. Telemetry could transmit images from in vivo microdevices, allowing visualization of the functional condition of internal organs.

What will be new in 2050 is that all essential physiological variables will be monitored throughout the patient continuously in time, thereby mapping integrally the temporal and spatial (patho)physiological status of the patient. Concepts such as feedback and set point will be developed to take the complexity of the critically ill patient into account and be multivariate and changing in time. In addition biosensors will be optimized in number, parameters, and location to provide essential information to drive therapeutic theragnostic [12] modalities normalization or organ function and outcome. Such information will also control and support implantable artificial organs and organ assist devices. Currently placed ex vivo devices such as continuous venovenous hemofiltration, extracorporeal membrane oxygenation, and mechanical ventilation will be placed in vivo and form part of the physiology of the patient in the ICU of 2050 [13]. The sensors of the ICU in vivo will generate massive amounts of information which will be fed into a time variant self-learning 
physiological model of the patient $[14,15]$. Such a "virtual patient" which will form the ex vivo platform for the control of the patient by the intensivist (Fig. 1).

There will be those that may remember the 1966 cult film The Fantastic Voyage, in which Stephen Boyd, Raquel Welch, and Donald Pleasence play medical doctors of the future and treat an incurable critically ill patient by entering a submarine that becomes miniaturized and injected into the circulation of the patient. Although the miniaturization of intensivists is not envisaged in the foreseeable future, many if not most of the elements of the ICU that are currently placed outside of the patients will, in my view, be administered in vivo. In this context a better paradigm for predicting the future ICU of 2050 than the saying of Niels Bohr quoted at the beginning of this paper would be that of Alan Kay who said that "the best way to predict the future is to invent it." It will be up to the intensivists, the scientific community, and ultimately to industry about whether the ICU in vivo will indeed be a reality. Only the future will tell.

\section{Author details}

${ }^{1}$ Department of Intensive Care, Erasmus MC, University Medical Center Rotterdam, 's-Gravendijkwal 230, 3015 CE Rotterdam, The Netherlands. ${ }^{2}$ Department of Translational Physiology, Academic Medical Center, University of Amsterdam, Meibergdreef 9, 1105 AZ Amsterdam, The Netherlands.

\section{Acknowledgements}

The author wishes to thank Yasin Ince for making Fig. 1.

\section{Compliance with ethical standards}

\section{Conflicts of interest}

Dr. Ince runs an Internet site microcirculationacademy.org which offers services (e.g., training, courses, analysis) related to clinical microcirculation.

\section{Open Access}

This article is distributed under the terms of the Creative Commons Attribution-NonCommercial 4.0 International License (http://creativecommons.org/ licenses/by-nc/4.0/), which permits any noncommercial use, distribution, and reproduction in any medium, provided you give appropriate credit to the original author(s) and the source, provide a link to the Creative Commons license, and indicate if changes were made.
Received: 2 January 2017 Accepted: 17 April 2017

Published online: 8 May 2017

\section{References}

1. Marini JJ, Vincent JL, Annane D (2015) Critical care evidence-new directions. JAMA 313(9):893-894 (3)

2. Christaki E, Giamarellos-Bourboulis EJ (2014) The beginning of personalized medicine in sepsis: small steps to a bright future. Clin Genet 86:56-61

3. Okusa MD, Jaber BL, Doran P, Duranteau J, Yang L, Murray PT, Mehta RL, Ince C (2013) Physiological biomarkers of acute kidney injury: a conceptual approach to improving outcomes. Contrib Nephrol 182:65-81

4. Ferrer R, Artigas A (2011) A physiologic parameters as biomarkers: what can we learn from physiologic variables and variation? Crit Care Clin 27:229-240

5. Ince C (2017) Personalized physiological medicine. Crit Care (in press)

6. Ince C (2015) Hemodynamic coherence and the rationale for monitoring the microcirculation. Crit Care 19:S8-S13

7. Stone DJ, Celi LA, Csete M (2015) Engineering control into medicine. J Crit Care 30:652.e1-7

8. Luke HD (1999) The origins of the sampling theorem. IEEE Commun 37:106-108

9. Eckert MA, Vu PQ, Zhang K, Kang D, Ali MM, Xu C, Zhao W (2013) Novel molecular and nanosensors for in vivo sensing. Theranostics 3(8):583-594 (23)

10. Slomovic S, Pardee K, James J, Collins J (2015) Synthetic biology devices for in vitro and in vivo diagnostics. Proc Natl Acad Sci USA 112(47):1442914435 (24)

11. Chatupniak A, Morales-Narvaez E, Merkogi A (2015) Micro and nanomotors in diagnostics. Adv Drug Deliv Rev 1(95):104-116

12. Pene F, Courtine E, Cariou A, Mira JP (2009) Toward theragnostics. Crit Care Med 37(Suppl.):S50-S58

13. Attanasio C, Latancia MT, Otterbein LE, Netti PA (2016) Update on renal replacement therapy: implantable artificial devices and bioengineered organs. Tissue Eng Rev 22(4):330-340

14. Hunter P (2016) The virtual physiological human: the physiome project aims to develop reproducible, multiscale models for clinical practice. IEEE Pulse 7(4):36-42

15. Li XI, Wang Y (2016) Adaptive online monitoring for ICU patients by combining just-in-time learning and principal component analysis. J Clin Monit Comput 30(6):807-820 